\title{
A Sensitive Voltammetric Sensor Based on Au Nanoparticle Decorated Graphene Nanosheets Modified Glassy Carbon Electrode for Determination of Acetylcholine in Presence of Dopamine
}

\author{
Masoud Reza Shishehbore, Mohadeseh Safaei* \\ Department of Chemistry, Yazd Branch, Islamic Azad University, Yazd, Iran \\ E-mail: mohadesehsafaei2017@gmail.com, mohadeseh_safaei@yahoo.com
}

\begin{abstract}
In this study the electro oxidation of acetylcholine (ACh) in pH 7.0 phosphate buffer solution (PBS) was investigated using cyclic voltammetry $(\mathrm{CV})$ and differential pulse voltammetry (DPV) with the modified glassy carbon electrode (GCE) by Au nanoparticle decorated graphene nanosheets (Au NPs/GNs). DPV is a rapid and sensitive electro analytical technique for determination of acetylcholine in presence of dopamine (DA). Using DPV technique, the current was linear within a concentration range of 0.1-700.0 $\mu \mathrm{M}$ of acetylcholine. The detection limit of the method for acetylcholine is $0.04 \mu \mathrm{M}(\mathrm{S} / \mathrm{N}=3)$. Diffusion coefficient, $\mathrm{D}$, and charge transfer coefficients, $\alpha$, have been determined for oxidizing acetylcholine at the modified surface. The applicability of the proposed method was shown by the successful analysis of acetylcholine in real sample.
\end{abstract}

Keywords: Acetylcholine, Dopamine, Au NPs/GNs nanocomposite, Differential pulse voltammetry, Glassy carbon electrode

\section{Introduction}

Currently, many analytical instruments used in environmental, food, pharmaceutical or clinical laboratories and also most of the commercial point of care devices work using electrochemical sensors, as a whole or a basic part. Electrochemical sensors are considered reliable, selective and sensitive compared to alternative analytical tools, and also entail benefits such as simplicity, prompt response time and are also cost-efficient ${ }^{[1-3]}$. There are also some disadvantages concerning bare electrodes in that they restrict their implementation of simple, comparatively high-concentrated and clean systems i.e. without intervening electro-active species ${ }^{[4-6]}$. Thus, electro-activity and morphological characteristics modification of the electrode surface on the basis of various methods is proposed as a means of overcoming the restrictions. Hence, implementing nanoparticles is proposed as surface modifiers to improve the performance of electrodes ${ }^{[7,8]}$.

Graphene sheets offer extraordinary electronic, thermal, and mechanical properties and are expected to find a variety of applications, such as sensors, nanocomposites, batteries, supercapacitors, and hydrogen storage ${ }^{[9]}$. Graphene sheets, which possess unique nanostructure and a variety of fascinating properties, are considered as promising nanoscale building blocks of new nanocomposites, namely as a support material for the dispersion of metal nanoparticles ${ }^{[10,11]}$. Graphenebased/Au nanocomposites have been used in various applications. These nanocomposites combine the intrinsic properties of Au nanoparticles with those of graphene-based materials, such as high electrical conductivity and enhanced surface area, which are essential for the high sensitivity of biosensors ${ }^{[12-14]}$.

Acetylcholine (ACh), an organic chemical, is released by nerve cells to deliver signals to the other cell types and acts as a neurotransmitter. ACh and its metabolite choline play vital roles in brain chemistry. They are needed for three main physiological functions i.e. learning, memory and attention ${ }^{[15,16]}$. Inside the nervous system, ACh functions as neuromodulator in both peripheral nervous system and central nervous system. Since loss of neurotransmission and modulation are linked with ACh, the several neural disorders are therefore associated with ACh including Alzheimer's disease, Parkinson diseases, schizophrenia and progressive dementia. According to the cholinergic hypothesis, Alzheimer's disease is occurred due to reduced synthesis of the $\mathrm{Ach}^{[17-20]}$. Hence, the quantitative determination of ACh is very important in biological sciences and clinical analysis. Numerous methods have been designed to detect ACh, including high performance liquid chromatography ${ }^{[21]}$, gas chromatography mass spectrometry ${ }^{[2]}$, capillary zone electrophoresis

Copyright (C2020 Mohadeseh Safaei, et al.

DOI: https://doi.org/10.37256/nat.112020182.43-52

This is an open-access article distributed under a CC BY license

(Creative Commons Attribution 4.0 International License)

https://creativecommons.org/licenses/by/4.0/ 
${ }^{[23]}$, potentiometry ${ }^{[24]}$, colorimetry ${ }^{[25]}$, chemiluminescence ${ }^{[26]}$ and photoelectrochemistry ${ }^{[27]}$. Specifically speaking, electrochemical techniques are mainly based on analyzing the relationship between determine and current, voltage or resistance. The emergence of new materials, makes it possible to build new electrochemical sensors with enhanced sensitivity and stability, which offer advantages like fast response, good portability, ease of operation, low cost and higher accuracy and low detection limit, as compared with the other analytical techniques ${ }^{[28,29]}$.

Dopamine is vital for coordinated motion and for association learning linked to behavioral reinforcement. Dopaminergic mechanisms of the striatum are intimately involved in motor coordination, complex issues of behavioral reinforcement, and disorders such as schizophrenia and Parkinson's disease $\mathrm{e}^{[30-32]}$. The striatum receives the densest dopaminergic innervation in the mammalian brain, which arises from neurons located in the substantia nigra and ventral tegmental area of the midbrain. In addition, the striatum is densely innervated by local cholinergic interneurons that are topically active and release acetylcholine. Histochemical studies have indicated that nicotinic acetylcholine receptors are expressed on dopaminergic nerve terminals in the striatum ${ }^{[33-35]}$.

In this study, production, characterization, and analytical performance of the sensor for acetylcholine is presented of dopamine including $\mathrm{Au} \mathrm{NPs} / \mathrm{GNs}$ nanocomposite on the surface of a GCE. Control of the proposed sensing method was made on real samples to be applied in the determination of acetylcholine for its critical concentration range.

\section{Experimental}

\subsection{Apparatus}

SEM measurements have been done with a Mira 3-XMU field emission SEM at an acceleration voltage of 15.0 $\mathrm{kV}$. KBr discks on a JASCO FT/IR-460 PLUS instrument was used to record FT-IR spectra. An Autolab potentiostat/ galvanostat (PGSTAT 302N, Eco Chemie, the Netherlands) was utilized to conduct electrochemical measurements. The General Purpose Electrochemical System (GPES) software was used to control the defined experimental settings. The three electro-chemical cell system are a Pt wire auxiliary electrode, a glassy carbon working electrode (GCE, modified or unmodified), and a saturated calomel reference electrode (SCE). In order to take PH measurements, a $710 \mathrm{pH}$ meter metrohm was used.

\subsection{Chemicals}

Acetylcholine (Sigma-Aldrich), dopamine and all other reagents were of the analytical grade, and they were obtained from Merck (Darmstadt, Germany). The buffer solutions were prepared from orthophosphoric acid and its salts over the $\mathrm{pH}$ range of 2.0-9.0.

\subsection{Synthesis of Au NPs/GNs nanocomposite}

$\mathrm{GO}$ is synthesized from natural graphite using a Hummers method ${ }^{[36]} \mathrm{At}$ first, $1.0 \mathrm{~g}$ of graphite, $5.0 \mathrm{~g}$ of $\mathrm{NaNO}_{3}$ and 5.0 $\mathrm{g}$ of $\mathrm{KMnO}_{4}$ have been mixed and $50 \mathrm{~mL}$ of $\mathrm{H}_{2} \mathrm{SO}_{4}$ were added to the mixture at $0^{\circ} \mathrm{C}$. The suspension was then diluted with $100 \mathrm{~mL}$ water and reaction temperature is quickly enhanced by $90^{\circ} \mathrm{C}$ and maintained for 15 minutes which leads to a color change to brown. Afterward, the mixture was cooled down to $40^{\circ} \mathrm{C}$ for 30 minutes. Subsequently $50 \mathrm{~mL}$ of $\mathrm{H}_{2} \mathrm{O}_{2}$ aqueous solution was added to the mixture until its color change to yellow and then it was cooled down to room temperature. Then, it has been washed by rinsing and centrifuging with $5 \% \mathrm{HCl}$ and $\mathrm{H}_{2} \mathrm{O}$ several times. The final stable and brownish GO aqueous solution has been reduced by ascorbic acid ${ }^{[37]} .50 \mathrm{~mL} 20 \%$ ascorbic acid has been poured to $50 \mathrm{~mL}(0.1 \mathrm{mg}$ $\mathrm{mL}-1$ ) of an aqueous dispersion of $\mathrm{GO}$ via robust shaking. After adding $50 \mathrm{~mL} \mathrm{H}_{2} \mathrm{O}$, the mix was maintained for $12 \mathrm{~h}$. The resulting graphene was rinsed by $\mathrm{H}_{2} \mathrm{O}$ and dried in $50^{\circ} \mathrm{C}$ before use. For synthesis of Au NPs, $2 \mathrm{~mL}$ of $5.0 \mathrm{mM} \mathrm{HAuCl}_{4}$ was heated and $0.5 \%$ sodium citrate was added dropwise at the boiling point and continues to heat until the color change to wine $\mathrm{red}^{[38]}$. In order to synthesis of Au NPs/GNs nanocomposite, $1.0 \mathrm{~g}$ GNs was added to $25.0 \mathrm{~mL} \mathrm{Au} \mathrm{NPs} \mathrm{solution} \mathrm{and}$ then was stirred for $10 \mathrm{~min}$ and then filtrated. Then, it has been dried at room temperature.

\subsection{Preparation of the electrode}

Before the surface was modified, the GCE was cautiously cleansed using $0.3 \mu \mathrm{m}$ and $0.05 \mu \mathrm{m}$ alumina slurries to acquire a surface resembling a mirror. The electrode was rinsed using water upon sonication in water and ethanol continuously for $20 \mathrm{~s}$. Au NPs/GNs nanocomposite has been used for covering unmodulated GCE. An Au NPs/ GNs nanocomposite stock solution within $1 \mathrm{~mL}$ aqueous solution has been collected by scattering $1 \mathrm{mg} \mathrm{Au} \mathrm{NPs/GNs}$ nanocomposite and ultrasonicating for 1 hour, and a $4 \mu \mathrm{l}$ aliquot of the $\mathrm{Au} \mathrm{NPs} / \mathrm{GNs} / \mathrm{H}_{2} \mathrm{O}$ suspension solution has been inserted on these carbon working electrodes. The resulting product has been put aside, and the solvent has been evaporated within room temperature. 


\subsection{Preparation of real samples}

Upon collecting urine samples, they were promptly kept in a refrigerator. 15 minute at $2000 \mathrm{rpm}$ centrifugation was implemented for $10 \mathrm{ml}$ of the samples. A $0.45 \mu \mathrm{m}$ filter was used to filter the supernatant. Then, various solution volumes were put into a $25 \mathrm{ml}$ volumetric flask prior to being diluted with PBS of pH 7.0 to the mark. Various volumes of acetylcholine and dopamine were used to spike the diluted urine samples. The proposed method was used to analyse the acetylcholine and dopamine contents via the standard addition method.

The sample of the acetylcholine and dopamine ampule was prepared by $1 \mathrm{ml}$ acetylcholine and dopamine ampoule has been diluted to $10 \mathrm{~mL}$ with $0.1 \mathrm{M}$ PBS (pH 7.0). Next, distinct volumes of the diluted solution have been poured into a $25 \mathrm{~mL}$ volumetric flask and diluted to the mark with PBS pH 7.0. The supposed method has been employed for analyzing acetylcholine and dopamine content through the standard addition method.

\section{Result and discussion}

\subsection{Morphology and structure of Au NPs/GNs nanocomposite}

The characterization of GO before and after the reduction has been examined by Fourier transform infrared spectroscopy (FT-IR) and SEM. As for FT-IR spectra (Fig. 1), the peak at $3400 \mathrm{~cm}^{-1}$ and 2 peaks emerged at 1730, 1225 and $1050 \mathrm{~cm}^{-1}$ in the FT-IR spectrum of GO relative to $\mathrm{CO}$ stretching, $\mathrm{COH}$ stretching vibrations and $\mathrm{CO}$ alkoxy stretching vibration (Fig. 1A). After reduction by ascorbic acid, the adsorption peaks at 1730 and $1225 \mathrm{~cm}^{-1}$ almost vanished that indicates removing oxygenated groups from the GO surface and largely leaves residual COC groups. Strengthening and broadening of the peak at $1550 \mathrm{~cm}^{-1}$ represents a degree of disorder and may be associated with bending graphite sheets. The band at $1640 \mathrm{~cm}^{-1}$ may be allocated to skeletal vibrations of unoxidized graphitic domains (Fig. 1B).
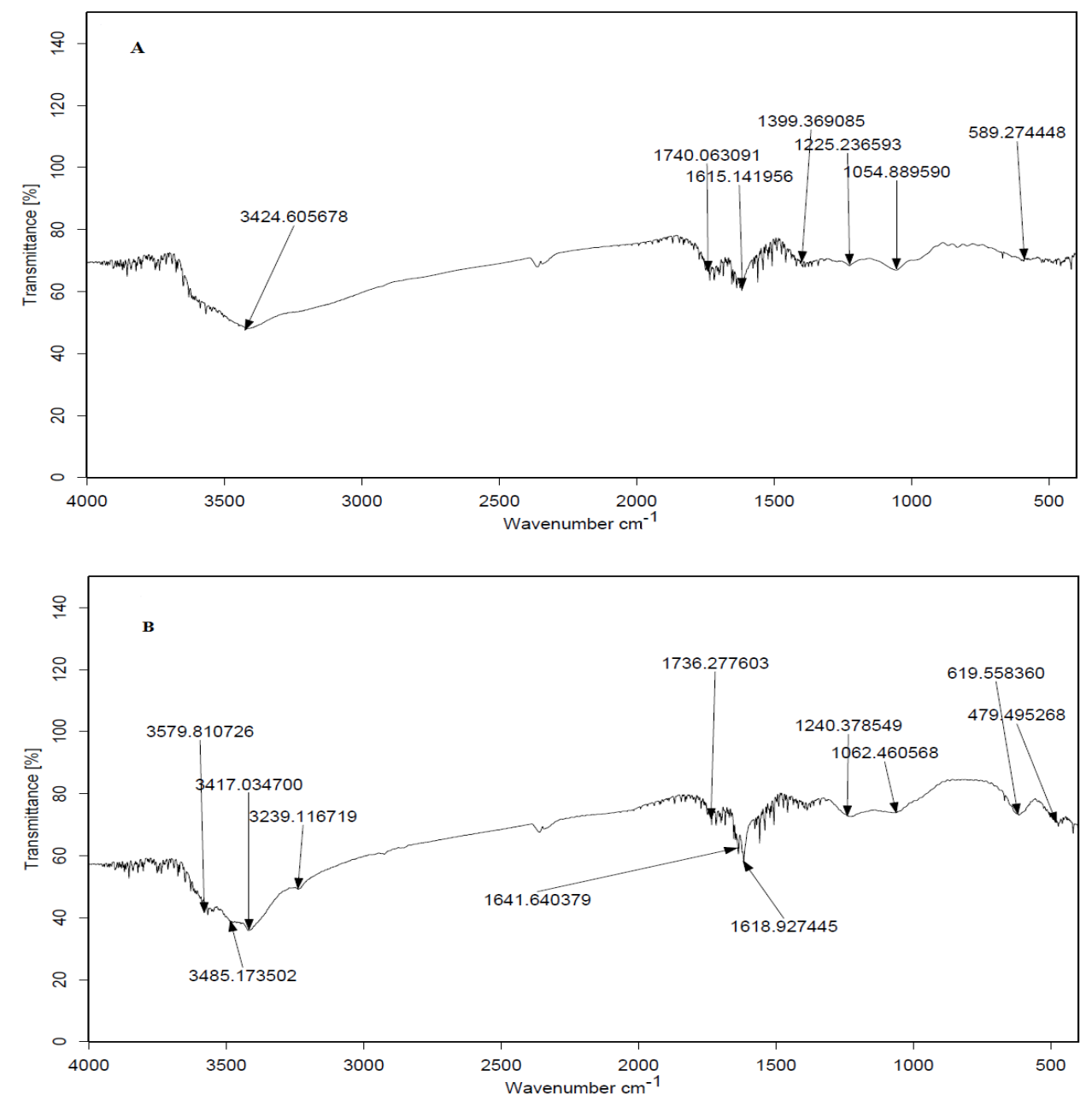

Figure 1. FTIR spectrum of GO (A) and GNs (B) 
SEM micrographs of the prepared GO, GNs and Au NPs are shown in Fig. 2. As it can be seen, GO and GNs contains thin sheets with random aggregation and crumple that is also observed with wrinkle and fold on GO surface (Fig. 2A) and GNs (Fig. 2B). Moreover, the SEM image of Au NPs/GNs displays that Au NPs are distributed evenly on the surface of GNs (Fig. 2C). Au NPs with a diameter of $<20 \mathrm{~nm}$ are homogeneously distributed in the GNs.
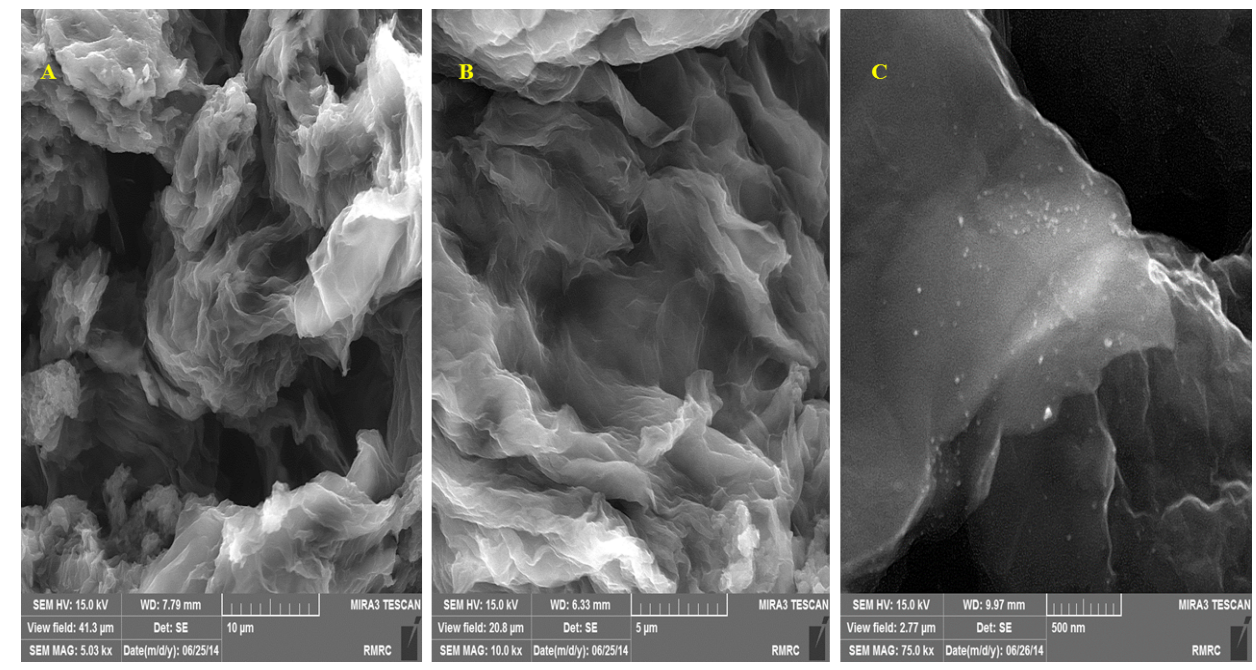

Figure 2. SEM image of GO (A), GNs (B) and Au NPs/GNs (C)

\subsection{Electrochemical behaviour of acetylcholine at the surface of various electrodes}

Acetylcholine electrochemical activities are dependent on the aqueous solution's $\mathrm{pH}$ value. Thus, the solution $\mathrm{pH}$ optimization is vital to acquire favorable results for acetylcholine electro-oxidation. Moreover, acetylcholine electrochemical behavior was examined via a $0.1 \mathrm{M}$ phosphate buffer solution (PBS) in various $\mathrm{pH}$ values in the 2.0-9.0 range by voltammetry at $\mathrm{Au} \mathrm{NPs/GNs/GCE} \mathrm{surface.} \mathrm{Results} \mathrm{indicated} \mathrm{that} \mathrm{acetylcholine} \mathrm{electro-oxidation} \mathrm{at} \mathrm{Au} \mathrm{NPs/GNs/}$ GCE surface is more favourable under neutral circumstances compared to acidic or basic medium state. The optimal pH for acetylcholine at was selected at $\mathrm{pH} 7.0$ at $\mathrm{Au}$ NPs/GNs/GCE surface. 100.0 $\mu \mathrm{M}$ acetylcholine oxidation at the Au NPs/ GNs/GCE (Curve c), GNs/GCE (Curve b) and unmodified GCE (Curve a) are illustrated in Fig.3.

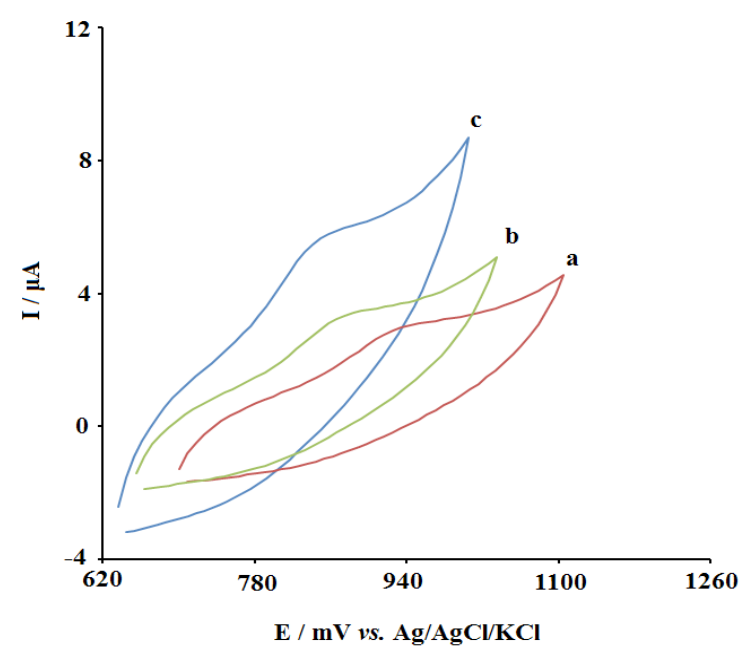

Figure 3. CVs of a) unmodified GCE, b) GNs/GCE and c) Au NPs/GNs/GCE in the presence of 100.0 $\mu$ M of acetylcholine at pH 7.0. In all cases, the scan rate was $100 \mathrm{mV} \mathrm{s}^{-1}$

Because of acetylcholine oxidation, that is approximately $80 \mathrm{mV}$ more negative compared to the unmodified GCE, the peak potential happens at $840 \mathrm{mV}$. Furthermore, regarding acetylcholine oxidation, Au NPs/GNs/GCE exhibits more anodic peak current in comparison to unmodified Au NPs/GNs /GCE which is a sign that unmodified GCE modification with $\mathrm{Au}$ NPs/GNs has considerably enhanced electrode performance towards acetylcholine oxidation. Increasing electro- 
chemical catalytic activities of $\mathrm{Au}$ NPs/GNs/GCE caused by introducing Au NPs/GNs can be related to the augmented surface area supplied by Au NPs/GNs and significant electron-transfer capability of nano-materials. Additionally, Au NPs/ GNs exhibit higher level of conductivity, acceptable bio-compatibility, and rapid electron-transfer abilities. Thus, Au NPs/ GNs/GCE significantly increased transferring electron acetylcholine.

\subsection{Effect of scan rate}

The scan rate impacts on the oxidation peak current of acetylcholine have been examined on the Au NPs/GNs/ GCE via cyclic voltammetry. As seen in Figure 4, intensity of the peak current is directly proportionate to the scan rate; that is, enhancement of the scan rate is followed by the increased peak current intensity. In addition, current is directly proportionate to the scan rate square root at a range of 30 to $700 \mathrm{mVs}^{-1}$ (Fig. 4A) that is an influential implication that diffusion controls the acetylcholine redox reaction.

Tafel plots were drawn from the data of the rising part of the current-voltage curves recorded at a scan rate of $10 \mathrm{mVs}$ ${ }^{1}$ for acetylcholine. Tafel slope of $0.1965 \mathrm{~V}$ was obtained, which agree well with the involvement of one electron at the rate determining step of the electrode process, ${ }^{[39]}$ assuming charge transfer coefficients $\alpha=0.6$ for acetylcholine (Fig. 4B).

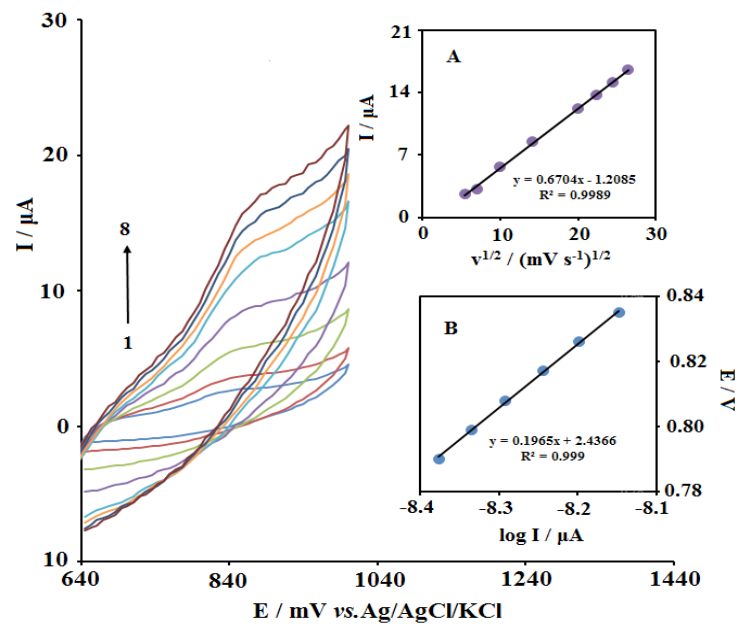

Figure 4. CVs of Au NPs/GNs/GCE in 0.1 M PBS (pH 7.0) containing $100.0 \mu \mathrm{M}$ of acetylcholine at various scan rates; numbers 1-8 correspond to $30,50,100,200,400,500,600$, and $700 \mathrm{mV} \mathrm{s}^{-1}$, respectively. Insets: (A) variation of anodic peak current vs. square root of scan rate and (B) Tafel plot derived from the $\mathrm{CV}$ at the scan rate of $10 \mathrm{mV} \mathrm{s}$

\subsection{Chronoamperometric measurements}

Acetylcholine at $\mathrm{Au} \mathrm{NPs} / \mathrm{GNs} / \mathrm{GCE}$ chronoamperometric measurements was conducted by modifying the working electrode potential at $890 \mathrm{mV}$ vs. $\mathrm{Ag} / \mathrm{AgCl} / \mathrm{KCl}(3.0 \mathrm{M})$ for different acetylcholine concentrations as depicted in Fig.5 at PBS (pH 7.0), respectively. Regarding electro-active materials, in this case acetylcholine, with D as the diffusion coefficient, thus electrochemical reaction current at mass transport limited status is presented by the Cottrell equation ${ }^{\text {[39] }}$.

$$
\mathrm{I}=\mathrm{nFAD}{ }^{1 / 2} \mathrm{C}_{\mathrm{b}} \pi^{-1 / 2} \mathrm{t}^{-1 / 2}
$$

According to Eq. (1), D ( $\left.\mathrm{cm}^{2} \mathrm{~s}^{-1}\right)$ represents analyte diffusion coefficient, F indicated Faraday constant (96485 CM), $\mathrm{C}_{\mathrm{b}}$ shows the analyte bulk concentration $\left(\mathrm{mol} \mathrm{cm} \mathrm{cm}^{-3}\right), \mathrm{n}$ represents the number of electrons exchanged per reactant molecule, and $\mathrm{A}$ is the electrode geometric area $\left(\mathrm{cm}^{2}\right)$. A linear curve has been obtained from the raw chronoamperometric traces for different concentrations of acetylcholinein through drawing I versus $\mathrm{t}^{-1 / 2}$ (Fig. $5 \mathrm{~A}$ ). Then, the slopes of the achieved direct lines versus acetylcholinein concentrations have been drawn (Fig. 5 B). Next, estimation of diffusion coefficient of $1.34 \times 10^{-6}$ $\mathrm{cm}^{2} \mathrm{~s}^{-1}$ has been performed for acetylcholinein. 


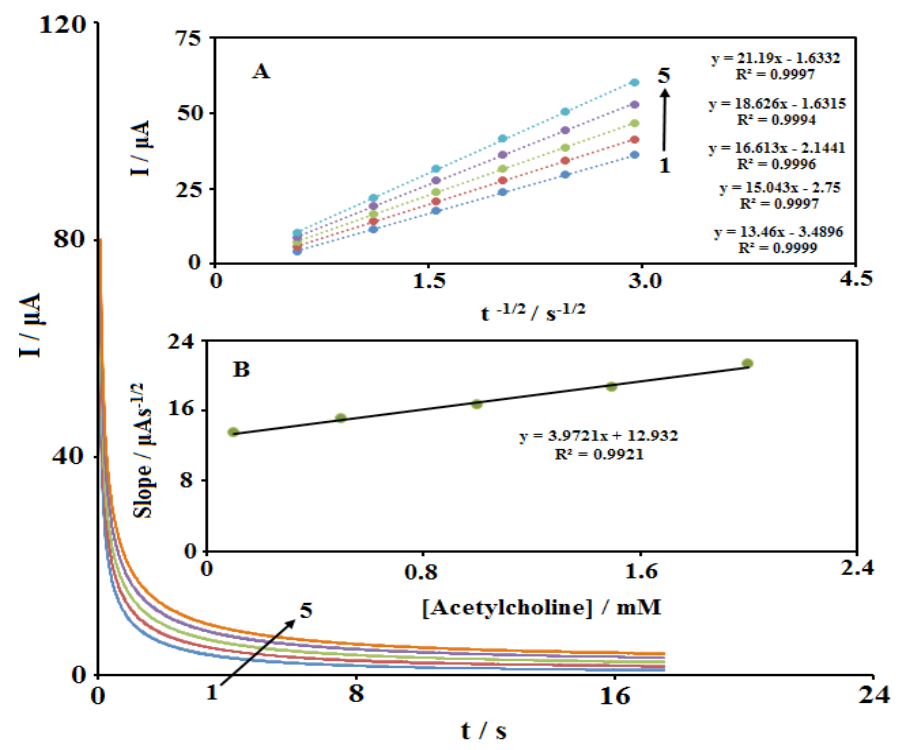

Figure 5. Chronoamperograms obtained at Au NPs/GNs/GCE in 0.1 M PBS (pH 7.0) for different concentrations of acetylcholine. The numbers 1-5 correspond to $0.1,0.5,1.0,1.5$ and $2.0 \mathrm{mM}$ of acetylcholine. Insets: (A) Plots of $I$ vs. $\mathrm{t}^{-1 / 2}$ obtained from chronoamperograms 1-5. (B) Plot of the slope of the straight lines against acetylcholine concentrations

\subsection{Calibration plot and limit of detection}

Acetylcholineat electro-oxidation peak currents at Au NPs/GNs/GCE surface can be utilized for acetylcholine determination within the solution. The benefit of DPV, differential pulse voltammetry is possessing enhanced sensitivity and features for analytical uses. By the use of Au NPs/GNs/GCE in 0.1 M PBS possessing different acetylcholine concentrations, DPV experimentation was conducted as presented in Fig.6. Results indicate that for acetylcholine oxidation electro-catalytic peak currents at $\mathrm{Au} \mathrm{NPs/GNs/GCE} \mathrm{surface,} \mathrm{there} \mathrm{is} \mathrm{linear} \mathrm{dependency} \mathrm{on} \mathrm{acetylcholine} \mathrm{concentrations}$ with then range of $0.1-700.0 \mu \mathrm{M}$ with 0.999 correlation coefficient. The acquired detection limit $(3 \sigma)$ was $0.04 \mu \mathrm{M}$.

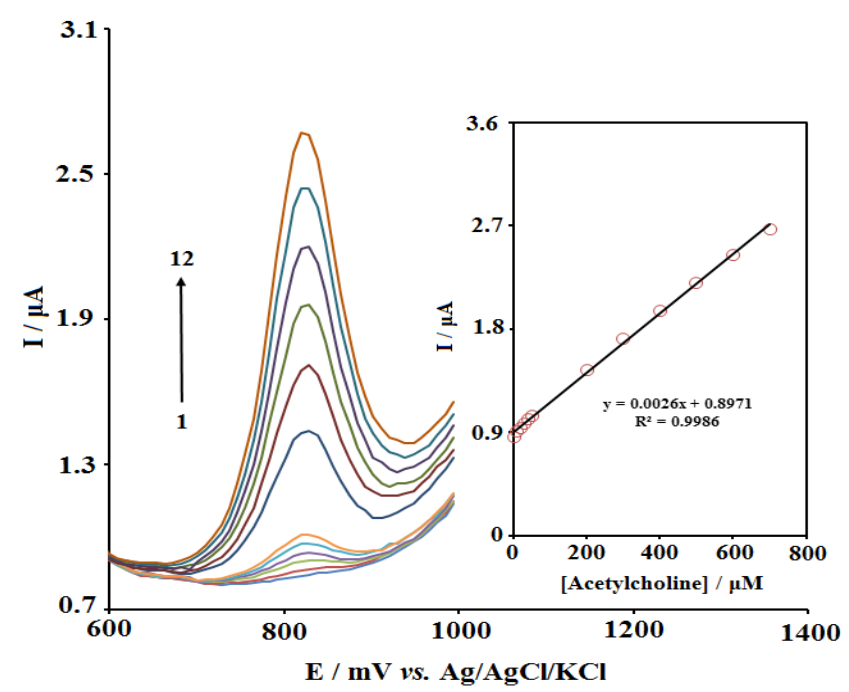

Figure 6. DPVs of Au NPs/GNs/GCE in 0.1 M PBS (pH 7.0) containing different concentrations of acetylcholine. Numbers 1-12 correspond to $0.1,10.0,20.0,30.0,40.0,50.0,200.0,300.0,400.0,500.0,600.0$ and $700.0 \mu \mathrm{M}$ of acetylcholine. The inset shows the plot of the peak current as a function of the acetylcholine concentration in the range of 0.1-700.0 $\mu \mathrm{M}$

\subsection{Determination of acetylcholine in the presence of dopamine}

To our knowledge, no paper has used NPs/GNs/GCE for determination of acetylcholine in the presence of dopamine and this is the first report for determination of acetylcholine in the presence of dopamine by using NPs/GNs/GCE. The two compounds were determined by simultaneously changing the concentrations of acetylcholine and dopamine, and recording the DPVs (Fig. 7). The voltammetric results showed well defined anodic peaks at potentials of 160 and $850 \mathrm{mV}$, 
corresponding to the oxidation of acetylcholine and dopamine, respectively, there by indicating that determination of these compounds is feasible at NPs/GNs/GCE, as shown in Fig. 7.

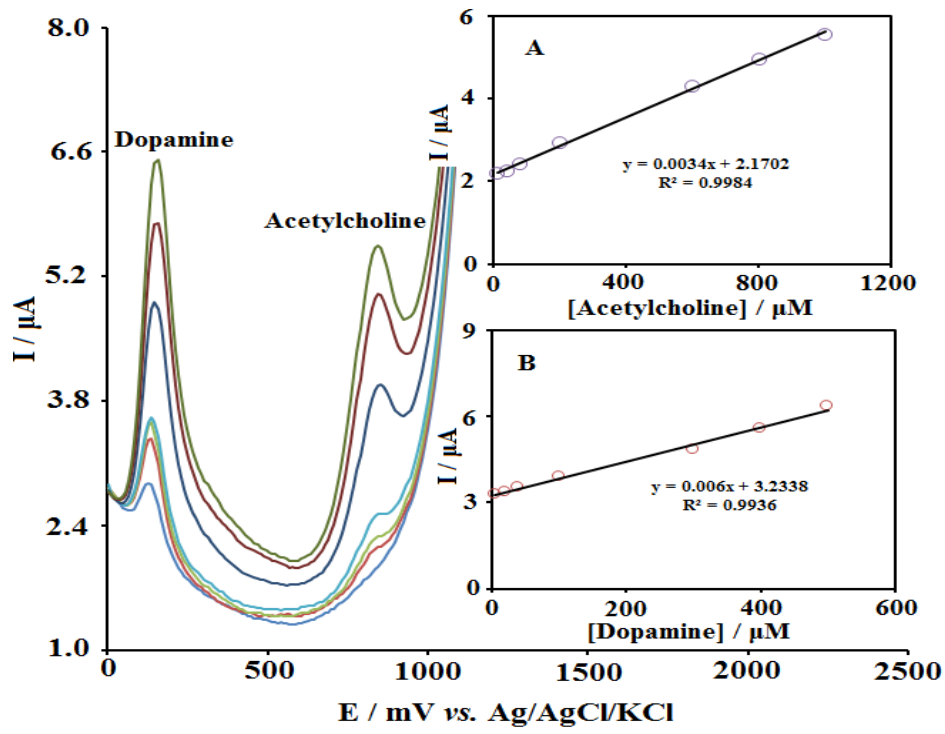

Figure 7. DPVs obtained at the surface of Au NPs/GNs/GCE in 0.1 M PBS (pH 7.0) containing different concentrations of acetylcholine and dopamine. DPVs from inner to outer correspond to $10.0+5.0,40.0+20.0,80.0+40.0,200.0+100.0,600.0+300.0,800+400.0$ and $1000.0+500.0 \mu M \quad$ of acetylcholine and dopamine respectively. Insets: (A) plot of $I_{\mathrm{pa}}$ vs. acetylcholine concentrations and (B) plot of $I_{\mathrm{pa}}$ vs. dopamine concentrations

\subsection{The repeatability and stability of Au NPs/GNs/GCE}

$\mathrm{Au} \mathrm{NPs/GNs/GCE} \mathrm{long} \mathrm{term} \mathrm{stability} \mathrm{was} \mathrm{assessed} \mathrm{for} \mathrm{a} \mathrm{period} \mathrm{of} \mathrm{three} \mathrm{weeks.} \mathrm{Prior} \mathrm{to} \mathrm{not} \mathrm{using} \mathrm{the} \mathrm{modified}$ electrode for three weeks while being stored at atmosphere, the tests were repeated. Based on cyclic voltammograms, there were no changes in acetylcholine oxidation peak potential with the exception of a reduction of less than $2.4 \%$ in comparison to first response. The modified electrode's antifouling characteristics regarding the oxidation of acetylcholine and relevant oxidation products were examined by recording CVs. At the vicinity of acetylcholine, upon potential cycling for 30 times at $50 \mathrm{mV} \mathrm{s}^{-1}$ scan rate, voltammograms were recorded. By examining the results, it is evident that there are no changes in peak potentials while there is a current reduction of less than $2.3 \%$. Based on these results, by applying a modified Au NPs/GNs/GCE, there will be enhanced sensitivity and a reduction in analyte and relevant oxidation product fouling effects.

\subsection{Real sample analysis}

For demonstrating practical uses of the method presented here, acetylcholine and dopamine have been detected in acetylcholine ampoule, dopamine ampoule and urine samples at Au NPs/GNs/GCE. All the samples have been treated in accordance with the section acetylcholine and dopamine voltammetric detection. Five parallel detections have been done for the samples. Table 1 reports the results. Recoveries have been $98.0 \%$ to $102.3 \%$ and the RSD (\%) has been less than 3.4 $\%$. Findings confirmed usability of the method recommended in real specimens. 
Table 1. Determination of acetylcholine and dopamine in acetylcholine ampoule, dopamine ampoule and urine samples. All the concentrations are in $\mu M(n=5)$

\begin{tabular}{|c|c|c|c|c|c|c|c|c|}
\hline \multirow[b]{2}{*}{ Sample } & \multicolumn{2}{|c|}{ Spiked } & \multicolumn{2}{|c|}{ Found } & \multicolumn{2}{|c|}{ Recovery (\%) } & \multicolumn{2}{|c|}{ R.S.D. (\%) } \\
\hline & acetylcholine & dopamine & acetylcholine & dopamine & acetylcholine & dopamine & acetylcholine & dopamine \\
\hline \multirow{3}{*}{ Acetylcholine } & - & - & 7.5 & $<\mathrm{DL}$ & - & - & 2.8 & - \\
\hline & 2.5 & 5.0 & 10.1 & 4.9 & 101.0 & 98.0 & 3.3 & 1.8 \\
\hline & 7.5 & 10.0 & 14.9 & 9.9 & 99.3 & 99 & 2.5 & 2.1 \\
\hline \multirow[t]{2}{*}{ ampoule } & 12.5 & 15.0 & 20.3 & 15.3 & 101.5 & 102.0 & 3.1 & 2.2 \\
\hline & - & - & - & 2.5 & - & - & - & 2.5 \\
\hline \multirow{2}{*}{ Dopamine } & 7.0 & 5.5 & 6.9 & 8.3 & 98.5 & 103.7 & 2.8 & 2.7 \\
\hline & 12 & 10.5 & 12.2 & 12.8 & 101.6 & 98.4 & 2.1 & 3.0 \\
\hline \multirow[t]{3}{*}{ ampoule } & 17.0 & 15.5 & 17.2 & 18.1 & 101.1 & 100.5 & 2.9 & 3.4 \\
\hline & - & - & $<\mathrm{DL}$ & $<\mathrm{DL}$ & - & - & - & - \\
\hline & 5.0 & 10.0 & 5.1 & 9.9 & 102.0 & 99.0 & 2.6 & 1.7 \\
\hline \multirow[t]{2}{*}{ Urine } & 10.0 & 20.0 & 9.8 & 20.3 & 98.0 & 101.5 & 2.7 & 2.4 \\
\hline & 15.0 & 30.0 & 14.8 & 30.7 & 98.6 & 102.3 & 2.2 & 2.3 \\
\hline
\end{tabular}

\section{Conclusions}

We have successfully synthesized Au NPs/GNs modified glassy carbon electrode. A rapid, simple, and sensitive method has been developed, optimized, and validated for the determination of acetylcholine in presence of dopamine. Hence the sensor can be effectively used for clinical analysis in the case where the levels of acetylcholine significantly increase. The proposed method provides significant advantages with satisfactory results and high recoveries.

\section{References}

[1] Geto A, Noori J S, Mortensen J, Svendsen W E, Dimaki M. Electrochemical determination of bentazone using simple screen-printed carbon electrodes. Environment international. 2019; 129: 400-407.

[2] Yadav M, Ganesan V, Gupta R, Yadav D K, Sonkar P K. Cobalt oxide nanocrystals anchored on graphene sheets for electrochemical determination of chloramphenicol. Microchemical Journal. 2019; 146: 881-887.

[3] Khorshed A A, Khairy M, Banks C E. Electrochemical determination of antihypertensive drugs by employing costless and portable unmodified screen-printed electrodes. Talanta. 2019; 198: 447-456.

[4] Zare H R, Shishehbore M R, Nematollahi D, Tehrani M S. Electrochemical behavior of nano-composite containing 4-hydroxy-2-(triphenylphosphonio) phenolate and multi-wall carbon nanotubes spiked in carbon paste and its application for electrocatalytic oxidation of hydrazine. Sensors and Actuators B: Chemical. 2010; 151(1): 153-161.

[5] Niedziałkowski P, Cebula Z, Malinowska N, Białobrzeska W, Sobaszek M, Ficek M, Ossowski T. Comparison of the paracetamol electrochemical determination using boron-doped diamond electrode and boron-doped carbon nanowalls. Biosensors and Bioelectronics. 2019; 126: 308-314.

[6] Qader B, Baron M, Hussain I, Sevilla J M, Johnson R P, Gonzalez-Rodriguez J. Electrochemical determination of disulfoton using a molecularly imprinted poly-phenol polymer. Electrochimica Acta. 2019; 295: 333-339.

[7] Zare H R, Shishehbore M R, Nematollahi D. A highly sensitive and selective sensor on the basis of 4-hydroxy-2(triphenylphosphonio) phenolate and multi-wall carbon nanotubes for electrocatalytic determination of folic acid in presence of ascorbic acid and uric acid. Electrochimica Acta. 2011; 58: 654-661.

[8] Safaei M, Beitollahi H, Shishehbore M R. Synthesis and characterization of $\mathrm{NiFe}_{2} \mathrm{O}_{4}$ nanoparticles using the hydrothermal method as magnetic catalysts for electrochemical detection of norepinephrine in the presence of folic acid. Journal of the Chinese Chemical Society. 2019; 66: 1-7.

[9] Mahmoudi E, Hajian A, Rezaei M, Afkhami A, Amine A, Bagheri H. A novel platform based on graphene nanoribbons/ protein capped $\mathrm{Au}-\mathrm{Cu}$ bimetallic nanoclusters: Application to the sensitive electrochemical determination of bisphenol A. Microchemical Journal. 2019; 145: 242-251.

[10] Cheong Y H, Nasir M Z M, Bakandritsos A, Pykal M, Jakubec P, Zbořil R, Pumera M. Cyanographene and graphene acid: the functional group of graphene derivative determines the application in electrochemical sensing and capacitors . ChemElectroChem. 2019; 6(1): 229-234.

[11] Afzali M, Mostafavi A, Shamspur T. Decoration of graphene oxide with NiO@ polypyrrole core-shell nanoparticles for the sensitive and selective electrochemical determination of piceatannol in grape skin and urine samples. Talanta. 2019; 196: 92-99.

[12] Sari T K, Takahashi F, Jin J, Zein R, Munaf E. Electrochemical determination of chromium (VI) in river water with gold nanoparticles-graphene nanocomposites modified electrodes. Analytical Sciences. 2018; 34(2): 155-160. 
[13] Li M, Wang W, Chen Z, Song Z, Luo X. Electrochemical determination of paracetamol based on Au@, graphene coreshell nanoparticles doped conducting polymer PEDOT nanocomposite. Sensors and Actuators B: Chemical. 2018; 260: 778-785.

[14] Yadav M, Ganesan V, Gupta R, Yadav D K, Sonkar P K. Cobalt oxide nanocrystals anchored on graphene sheets for electrochemical determination of chloramphenicol. Microchemical Journal. 2019; 146: 881-887.

[15] Martín-Barreiro A, de Marcos S, Jesús M, Grazú V, Galbán J. Gold nanocluster fluorescence as an indicator for optical enzymatic nanobiosensors: choline and acetylcholine determination. Sensors and Actuators B: Chemical. 2018; 277: 261-270.

[16] Balasubramanian P, Balamurugan T S T, Chen S M, Chen T W. Facile Synthesis of Spinel-Type Copper Cobaltite Nanoplates for Enhanced Electrocatalytic Detection of Acetylcholine. ACS Sustainable Chemistry \& Engineering. 2019; 7(8): 7642-7651.

[17] Giribaldi J, Dutertre S. $\alpha$-Conotoxins to explore the molecular, physiological and pathophysiological functions of neuronal nicotinic acetylcholine receptors. Neuroscience letters. 2018; 679: 24-34.

[18] Kong D, Jin R, Zhao X, Li H, Yan X, Liu F, Lu G. Protein-Inorganic Hybrid Nanoflower-Rooted Agarose Hydrogel Platform for Point-of-Care Detection of Acetylcholine. ACS applied materials \& interfaces. 2019; 11(12): 1185711864.

[19] Criado M. Acetylcholine nicotinic receptor subtypes in chromaffin cells. Pflügers Archiv-European Journal of Physiology. 2018; 470(1): 13-20.

[20] Chauhan N, Tiwari S, Narayan T, Jain U. Bienzymatic assembly formed@ Pt nano sensing framework detecting acetylcholine in aqueous phase. Applied Surface Science. 2019; 474: 154-160.

[21] Yoshida H, Yamada A, Todoroki K, Imakyure O, Nohta H, Yamaguchi M. Liquid chromatographic determination of acetylcholine based on pre-column alkaline cleavage reaction and post-column tris (2,2'-bipyridyl) ruthenium (III) chemiluminescence detection. Luminescence. 2009; 24: 306-310

[22] Patterson T A, Kosh J W. Simultaneous quantitation of arecoline, acetylcholine, and choline in tissue using gas chromatography/electron impact mass spectrometry. Biological Mass Spectrometry. 1992; 21: 299-304.

[23] Carter N, Trenerry V C. The determination of choline in vitamin preparations, infant formula and selected foods by capillary zone electrophoresis with indirect ultraviolet detection. Electrophoresis. 1996; 17: 1622-1626.

[24] Barsoum B N, Watson W M, Mahdi I M, Khalid E. Electrometric assay for the determination of acetylcholine using a sensitive sensor based on carbon paste. Journal of Electroanalytical Chemistry. 2004; 567: 277-281.

[25] Qian J, Yang X, Jiang L, Zhu C, Mao H, Wang K. Facile preparation of $\mathrm{Fe}_{3} \mathrm{O}_{4}$ nanospheres/reduced graphene oxide nanocomposites with high peroxidase-like activity for sensitive and selective colorimetric detection of acetylcholine. Sensors and Actuators B: Chemical. 2014; 201: 160-166.

[26] Buiculescu R, Hatzimarinaki M, Chaniotakis N. Biosilicated CdSe/ZnS quantum dots as photoluminescent transducers for acetylcholinesterase-based biosensors. Analytical and Bioanalytical Chemistry. 2010; 398: 3015-3021.

[27] Zayats M, Kharitonov AB, Pogorelova SP, Lioubashevski O, Katz E, Willner I. Probing photoelectrochemical processes in Au-CdS nanoparticle arrays by surface plasmon resonance: application for the detection of acetylcholine esterase inhibitors. Journal of the American Chemical Society. 2003; 125: 16006-16014

[28] Safaei M, Beitollahi H, Shishehbore M R, Tajik S. Electrocatalytic determination of captopril using a carbon paste electrode modified with $\mathrm{N}$-(ferrocenyl-methylidene) fluorene-2-amine and graphene/ZnO nanocomposite. Journal of the Serbian Chemical Society. 2019; 84(2): 175-185.

[29] Safaei M, Beitollahi H, Shishehbore M R. Simultaneous determination of epinephrine and folic acid using the $\mathrm{Fe}_{3} \mathrm{O}_{4} @$ $\mathrm{SiO}_{2} / \mathrm{GR}$ nanocomposite modified graphite. Russian Journal of Electrochemistry. 2018; 54(11): 851-859.

[30] Zhang W, Duan D, Liu S, Zhang Y, Leng L, Li X, Zhang Y. Metal-organic framework-based molecularly imprinted polymer as a high sensitive and selective hybrid for the determination of dopamine in injections and human serum samples. Biosensors and Bioelectronics. 2018; 118: 129-136.

[31] He S, He P, Zhang X, Zhang X, Liu K, Jia L, Dong F. Poly (glycine)/graphene oxide modified glassy carbon electrode: preparation, characterization and simultaneous electrochemical determination of dopamine, uric acid, guanine and adenine. Analytica Chimica Acta. 2018; 1031: 75-82.

[32] Liu X, Zhang W, Huang L, Hu N, Liu W, Liu Y, Wang J. Fluorometric determination of dopamine by using molybdenum disulfide quantum dots. Microchimica Acta. 2018; 185(4): 234-239.

[33] Wonnacott S, Bermudez I, Millar N S, Tzartos S J. Nicotinic acetylcholine receptors. British journal of pharmacology. 2018; 175(11): 1785-1788.

[34] Zoli M, Pucci S, Vilella A, Gotti C. Neuronal and extraneuronal nicotinic acetylcholine receptors. Current neuropharmacology. 2018; 16(4): 338-349.

[35] Jun H, Yu H, Gong J, Jiang J, Qiao X, Perkey E, Liu J. An immune-beige adipocyte communication via nicotinic acetylcholine receptor signaling. Nature medicine. 2018; 24(6): 814-821. 
[36] Hummers Jr W S, Offeman R E. Preparation of graphitic oxide. Journal of the American Chemical Society. 1958; 80(6): 1339-1339.

[37] Zhang J, Yang H, Shen G, Cheng P, Zhang J, Guo S. Reduction of graphene oxide via L-ascorbic acid. Chemical Communications. 2010; 46(7): 1112-1114.

[38] Rajeshwari A, Garg K, Elavarasi M, Chandrasekaran N, Mukherjee A. Interaction of citrate-capped gold nanoparticles with the selected amino thiols for sensing applications. Proceedings of the National Academy of Sciences, India Section B: Biological Sciences. 2017.p.23-30.

[39] Bard A, Faulkner L. Electrochemical methods fundamentals and applications, 2th ed. New York: Wiley; 2001. 